\title{
Rational design of a carboxylic esterase $R h$ Est1 based on computational analysis of substrate binding
}

Qi Chen ${ }^{1}$, Zheng-Jiao Luan ${ }^{1}$, Hui-Lei $\mathrm{Yu}^{1}$, Xiaolin Cheng ${ }^{2,3 *}$, Jian-He Xu ${ }^{1,4^{*}}$

${ }^{1}$ State Key Laboratory of Bioreactor Engineering, East China University of Science and Technology, Shanghai, China.

${ }^{2}$ UT/ORNL Center for Molecular Biophysics, Oak Ridge National Laboratory, Oak Ridge, Tennessee, United States.

${ }^{3}$ Department of Biochemistry and Cellular and Molecular Biology, University of Tennessee, Knoxville, Tennessee, United States.

${ }^{4}$ Shanghai Collaborative Innovation Center for Biomanufacturing, East China University of Science and Technology, Shanghai, China.

* Corresponding author:

Xiaolin Cheng

Oak Ridge National Laboratory, Center for Molecular Biophysics, P.O.Box 2008, Oak Ridge, TN, USA 37831. Fax: +1 865 5767651. Tel: +1 865 5760850. E-mail: chengx@ornl.gov.

Jian-He Xu

East China University of Science and Technology, State Key Laboratory of Bioreactor Engineering, 130 Meilong Road, Shanghai, CN 200237. Fax: +86 21 64250840. Tel: +86 21 64252498. E-mail: jianhexu@ecust.edu.cn. 


\section{Abstract}

A new carboxylic esterase RhEst1 which catalyzes the hydrolysis of $(S)-(+)$-2,2-dimethylcyclopropanecarboxylate $(S$-DmCpCe), the key chiral building block of cilastatin, was identified and subsequently crystallized in our previous work. Mutant $R h$ Est $_{\mathrm{A} 147 \mathrm{I} / \mathrm{V} 148 \mathrm{~F} / \mathrm{G} 254 \mathrm{~A}}$ was found to show a 5-fold increase in the catalytic activity. In this work, molecular dynamic simulations were performed to elucidate the molecular determinant of the enzyme activity. Our simulations show that the substrate binds much more strongly in the A147I/V148F/G254A mutant than in wild type, with more hydrogen bonds formed between the substrate and the catalytic triad and the oxyanion hole. The $\mathrm{OH}$ group of the catalytic residue Ser101 in the mutant is better positioned to initiate the nucleophilic attack on $S$-DmCpCe. Interestingly, the "170-179" loop which is involved in shaping the catalytic sites and facilitating the product release shows remarkable dynamic differences in the two systems. Based on the simulation results, six residues were identified as potential "hot-spots" for further experimental testing. Consequently, the G126S and R133L mutants show higher catalytic efficiency as compared with the wild type. This work provides molecular-level insights into the substrate binding mechanism of carboxylic esterase $R h$ Est1, facilitating future experimental efforts toward developing more efficient $R h$ Est1 variants for industrial applications.

Keywords: carboxylic esterase, molecular dynamics simulation, rational design, structural mechanism, catalytic efficiency 


\section{Introduction}

Cilastatin, a renal dehydropeptidase inhibitor, is one of the most popular drugs for antibiotic treatment. Industrial production of Cilastatin requires efficient synthesis of a chiral intermediate, ethyl $(S)-(+)-2,2$-dimethylcyclopropanecarboxylate (designated as $S$-DmCpCe hereafter) [1-3]. Compared with chemical synthesis methods, biocatalysis is a greener method due to its high efficiency, low cost and being environmentally friendly $[4,5]$.

Several efforts have been reported towards biocatalytic synthesis of $S$-DmCpCe using biotechnological processes [6-8]. Wang's group tested five commercial immobilized lipases from different organisms, and Novozym 435 was found to have high enantioselectivity and activity toward enzymatic resolution of racemic ( \pm -DmCpCe[9, 10]. Yeom et al. investigated the enantioselective production of (S)-2,2-dimethylcyclopropane carboxylic acid in 53 Rhodococcus and Pseudomonas related strains, and found that Rhodococcus erythropolis ATCC 25544 exhibited the highest enantioselectivity[11].

In our previous work, a carboxylic esterase $R h$ Est 1 was identified and isolated from Rhodococcus sp. ECU1013, which can catalyze the hydrolysis of $S$-DmCpCe with high enantioselectivity[12, 13] (Fig. S1). As the native enzyme exhibits relatively low activity to meet the industrial production requirements, both error-prone PCR and site-directed saturation mutagenesis have been applied to improve RhEstl's activity[14]. Towards a structure-based semi-rational design, the crystal structure of $R h$ Est1 was also determined at $1.9 \AA$ resolution (PDB ID: 4RNC)[14]. These efforts 
led to the discovery of a $R h \mathrm{Est}_{\mathrm{A} 147 \mathrm{I} / \mathrm{V} 148 \mathrm{~F} / \mathrm{G} 254 \mathrm{~A}}$ mutant that shows a 5-fold increase in the enzymatic activity compared to the native enzyme. However, this increased enzyme activity is still too low to be industrially viable for the production of $S$-DmCpCe. Further improvement in enzyme activity will require a deeper understanding of the molecular mechanism underlying how these mutations affect the enzyme activity.

Nowadays, structure-based molecular simulations have become a very useful tool for enzyme engineering[15-18], hinging on a detailed understanding of the substrate binding and catalytic mechanism. In this work, all-atom molecular dynamic (MD) simulations were performed to unravel molecular mechanism of the substrate binding and catalysis. Both the wild type (WT) and a triple mutant (A147I/V148F/G254A) $R h$ Est 1 with and without the substrate bound were simulated. From these simulations, crucial residues that might affect the esterase activity were identified and further subjected to site-directed mutagenesis to search for enzyme variants with improved catalytic efficiency.

\section{Materials and Methods}

\section{Computational methods}

The crystal structure of wild type $R h$ Est 1 has been resolved recently with $1.9 \AA$ resolution (PDB ID: 4RNC)[14]. The mutant structure $\left(\mathrm{APO}_{\mathrm{A} 147 \mathrm{I} / \mathrm{V} 148 \mathrm{~F} / \mathrm{G} 254 \mathrm{~A}}\right)$ was constructed via homology modeling method using the wild type crystal structure $\left(\mathrm{APO}_{\mathrm{WT}}\right)$ as a template. AutoDock Vina was then used to obtain starting structures of 
the wild type and A147I/V148F/G254A mutant RhEst1 in complex with the substrate ( $S$-DmCpCe)[19]. The best docked structure for each system was selected according to the catalytic mechanism reported and binding affinity estimated by AutoDock Vina, and was further validated by visual inspection. Totally, four $R h$ Est 1 structures were generated, two for the wild type and two for the A147//V148F/G254A mutant, in both apo and substrate bound forms.

All the MD simulations were performed with the NAMD package, with Amber FF99SB force field parameters[20, 21]. The force field parameters for $S$-DmCpCe were generated using the Antechamber package and the partial charges were calculated using the RESP method[22, 23]. The force field parameters for the substrate are listed in Table S1. Missing atoms and hydrogen atoms were added using the LEaP module in Amber11[24]. Sodium ions were added to neutralize each system. Periodic boundary conditions were applied. Each simulation system was solvated in a TIP3P water box with a thickness of at least $10 \AA$ from the protein on each side of the box[25]. In the simulations, the van der Waals (vdW) interactions were smoothly turned off between 10.5 and $12 \AA$ using a switching function. Long-range electrostatic interactions were computed using the particle-mesh Ewald (PME) method[26]. The total number of atoms in the four systems is about 40,000 each.

The structures were energy minimized for 20000 steps each. After minimization, all the simulation systems were heated gradually from $0 \mathrm{~K}$ to $300 \mathrm{~K}$ in the NVT ensemble by Langevin dynamics, increasing $10 \mathrm{~K}$ every 1000 steps. The NPT 
ensemble was used in the following 2 ns of equilibration and 50 ns production simulations. The integration step is $2 \mathrm{fs}$, and the trajectories were saved every 2 ps.

Several analysis methods were applied such as Root Mean Square Deviation (RMSD), Root Mean Square Fluctuation (RMSF), correlation matrix analysis, and binding free energy estimation using the Molecular Mechanics Poisson Boltzmann (Generalized Born) surface area (MM-PB(GB)/SA) method. RMSD and RMSF calculations were performed using the Gromacs 4.5.5 program[27]. The correlation matrices of the $\mathrm{C} \alpha$ atomic fluctuations were calculated using the $\mathrm{R}$ package Bio3D[28]. 12,500 frames retrieved from the last $25 \mathrm{~ns}$ MD trajectories were used for the calculation after removing the global translational and rotational motions of the protein. To remove weak correlations, cross-correlation coefficient value of 0.5 was chosen as the threshold value for visualization. The MM-PB(GB)/SA binding free energies were calculated using 1000 snapshots retrieved from the last $25 \mathrm{~ns}$ MD trajectories for all the simulated systems[29]. According to the MM-PB(GB)/SA algorithm, the total binding free energy change $\left(\Delta G_{\text {bind }}\right)$ is calculated as the free energies difference between the complex $\left(G_{\text {complex }}\right)$, the receptor $\left(G_{\text {receptor }}\right)$ and the ligand $\left(G_{\text {ligand }}\right)$. For each free energy term $(G)$, it is defined as the sum of the internal energy $\left(E_{\mathrm{MM}}\right)$ in the gas phase, the solvation free energy which consists of the electrostatic component $\left(G_{\mathrm{PB}}\right.$ or $\left.G_{\mathrm{GB}}\right)$ and the nonpolar term $\left(G_{\text {nonpolar }}\right)$, and the entropy part (TS). The entropic contribution to the binding free energy was ignored in our calculation. Visualization and images of the enzyme structures were prepared with $\operatorname{VMD}[30]$. 
The "SuSPect" online server was used to predict whether or not a single amino acid variation is likely to affect protein stability and enzyme activity[31]. 77 sequence-, structure- and systems biology-based features are used to train a support vector machine (SVM) to discriminate between favorable and neutral variants. SuSPect accepts a protein sequence as an input. In this study, the mutations were also mapped to known structures and homology models to make more reliable predictions.

\section{Experimental methods}

Site-directed mutations were constructed by Quick Change ${ }^{\circledR}$ Site-Directed Mutagenesis Kit, and the plasmid pET28a(+) containing the $R h$ Est 1 gene was used as the template. The primers used for mutations are listed in Table S2. The PCR products digested with DpnI were transformed into E. coli BL21 (DE3) for esterase expression. Cultivation of the recombinant E. coli cells expressing RhEst1 or its mutants and enzyme purification using $\mathrm{Ni}^{2+}$ affinity chromatography were performed as described previously[13, 32, 33].

The activity assay and the detailed procedure for product detection have also been reported previously[14]. Briefly, enzyme activity assay was performed in $500 \mu \mathrm{L}$ of reaction mixture, containing a certain enzyme (cell-free enzyme or purified enzyme) and $10 \mathrm{mM}( \pm)-\mathrm{DmCpCe}\left(10 \% \mathrm{v} / \mathrm{v}\right.$ DMSO), conducted at $30^{\circ} \mathrm{C}$ in a $1000 \mathrm{rpm}$ oscillator for 5 minutes. Protein concentration was determined by the Bradford method with bovine serum albumin as the standard. The product was acidified with $20 \% \mathrm{H}_{2} \mathrm{SO}_{4}$, extracted with $500 \mu \mathrm{L}$ of ethyl acetate (containing $0.2 \mathrm{mM}$ dodecane as an internal standard) and detected by GC (SHIMADZU GC-2014). One unit of 
enzyme activity was defined as the amount of enzyme releasing $1.0 \mu$ mol of $S$-DmCpCa per minute under the assay conditions.

The kinetic parameters were determined by measuring the activity under varied substrate concentrations $(0.1-10 \mathrm{mM})$. The Michaelis-Menten $\left(K_{\mathrm{m}}\right)$ constant and the maximal reaction rate $\left(V_{\max }\right)$ of the enzyme were calculated from Lineweaver-Burk plots.

\section{Results}

Fig. 1(a) and Fig. S2 shows that the RMSDs of all the four systems have reached a plateau during the simulations, and the final RMSD values for all the systems are below $2.5 \AA$ A. According to the RMSF results shown in Fig. 1(b), the secondary structure elements like $\alpha$ helices and $\beta$ strands exhibit small fluctuations, while several loop regions such as loop "60-69" and loop "170-179" show significant flexibility. As the loop " $170-179$ " is located near the binding pocket, the conformation and flexibility of this loop may directly affect the substrate binding, which will be discussed further below.
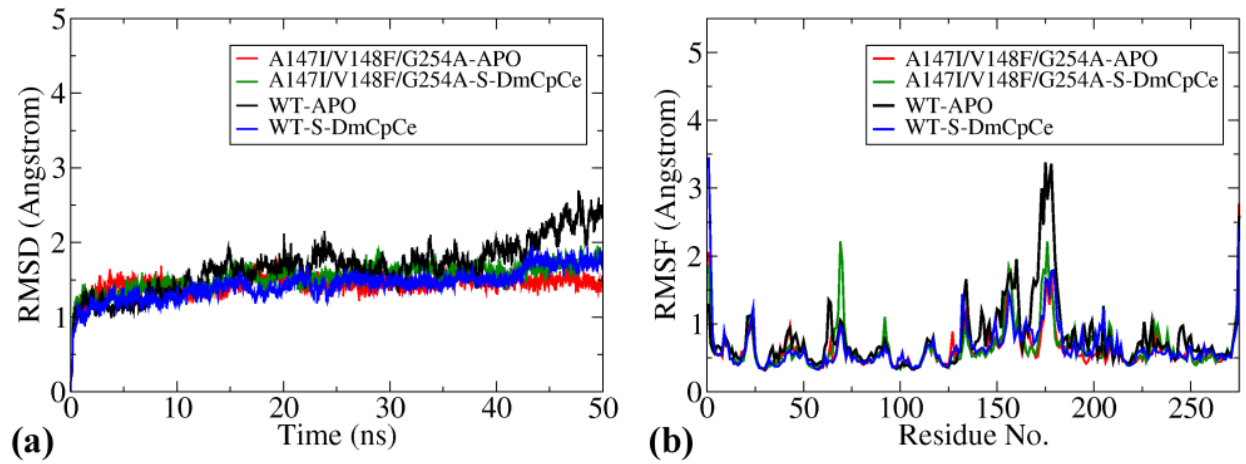

(b) 
Fig. 1. RMSD (a) and RMSF (b) results of WT and A147I/V148F/G254A mutant of $R h$ Est1 in apo form and in complex with $S$-DmCpCe, respectively.

\section{Binding mode analysis}

The crystal structure of the wild type $R h$ Est 1 was determined in our recent work $[34,35]$. $R h$ Est 1 belongs to the $\alpha / \beta$ hydrolase fold family and its catalytic triad was identified as Ser101, Asp225 and His253. According to the proposed catalytic mechanism of esterase (Fig. S3), the reaction is initiated by a nucleophilic attack of the carbonyl carbon by the $\mathrm{OH}$ group of Ser101, generating a tetrahedral intermediate. A low-barrier hydrogen bond is formed between Asp225 and His253 to facilitate the nucleophilic attack [36-38]. The polarized carbonyl oxygen atom is stabilized by an oxyanion hole involving the main chain nitrogen atoms of Trp33 and Tyr102.

From the simulations, the average distances between the carbonyl oxygen of $S$-DmCpCe and the main chain nitrogen atoms of $\operatorname{Trp} 33\left(\mathrm{~d}_{1}\right)$ and Tyr102 $\left(\mathrm{d}_{2}\right)$ are $2.2 \pm$ $0.2 \AA$ and $2.8 \pm 0.5 \AA$ respectively in wild type, $1.9 \pm 0.1 \AA$ and $3.0 \pm 0.4 \AA$ respectively in A147I/V148F/G254A mutant (Fig. 2 and Fig. S4). These results indicate that two strong hydrogen bonds are formed between the carbonyl $\mathrm{O}$ atom and the oxyanion hole to stabilize the substrate binding in both wild type and A147I/V148F/G254A mutant $R h$ Est1. However, the distance between the carbonyl carbon and the $\mathrm{OH}$ group of Ser101 $\left(\mathrm{d}_{3}\right)$ is found to be slightly shorter in the A147I/V148F/G254A mutant $(3.9 \pm 0.3 \AA)$ than in wild type $(4.4 \pm 0.3 \AA)$, and the distance between the ester oxygen atom of the substrate and the catalytic residue His $253\left(\mathrm{~d}_{4}\right)$ is also much shorter in the mutant $(3.2 \pm 0.6 \AA)$ than that in WT $(4.7 \pm 0.5$ 
$\AA$ ). Taken together, the substrate appears to form stronger interactions (hydrogen bonds) with the catalytic triad and the oxyanion hole in A147I/V148F/G254A mutant than in wild type. More importantly, the hydroxyl oxygen atom of Ser101 appears to be situated in a better orientation in the mutant to initiate the nucleophilic attack on the substrate. That is, in the mutant the substrate not only binds more strongly, but also is in a more active conformation with all catalytic site residues prearranged for the reaction.

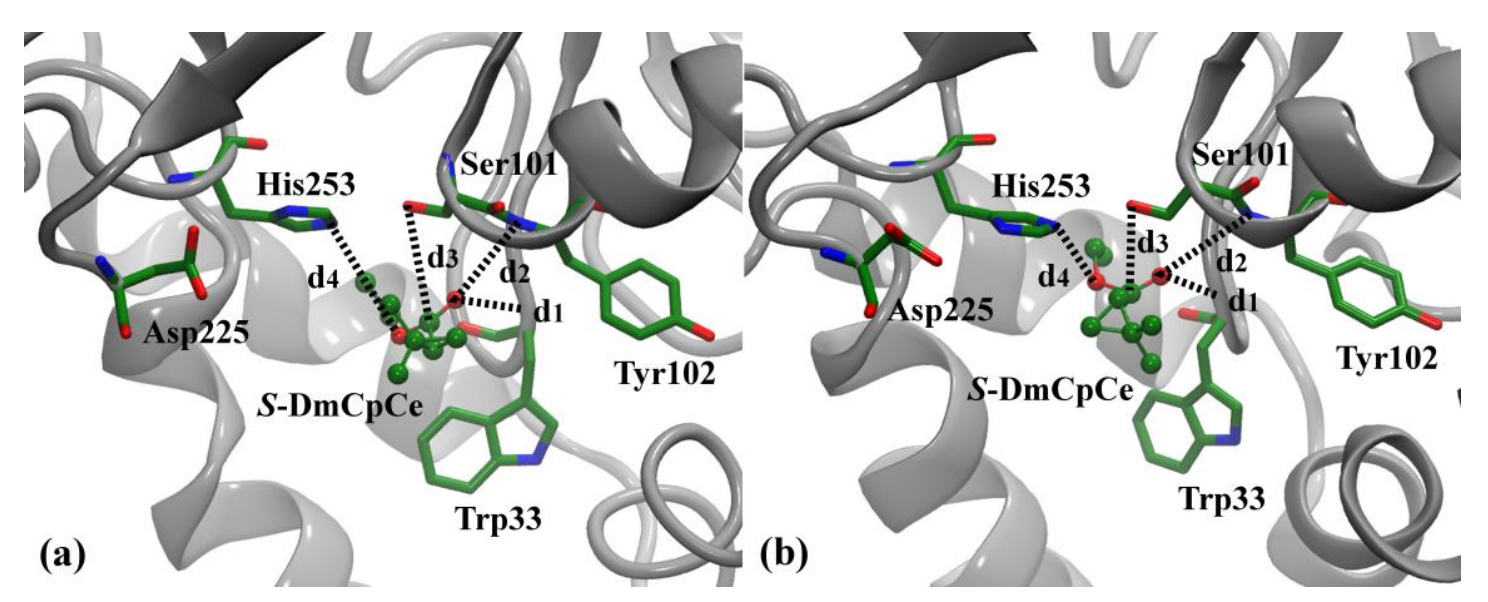

Fig. 2. Schematic diagram of the binding mode of $S$-DmCpCe in $R h \mathrm{Est}_{\mathrm{WT}}-S$-DmCpCe (a) and $R h \mathrm{Est}_{\mathrm{A} 147 \mathrm{I} / \mathrm{V} 148 \mathrm{~F} / \mathrm{G} 254 \mathrm{~A}}-S$-DmCpCe (b).

The activity of $R h \mathrm{Est}_{\mathrm{A} 147 \mathrm{I} / \mathrm{V} 148 \mathrm{~F} / \mathrm{G} 254 \mathrm{~A}}$ variant towards the hydrolysis of $S$-DmCpCe was found to be improved than that of the wild type using the error-prone PCR method in our previous work[14]. However, the mutated sites Ala147 and Val148 are both located near the substrate entrance channel but distant from the active site, whereas Gly254 is positioned near the catalytic triad His253. Fig. 3 shows an overview of the substrate binding poses in the two RhEst1 complexes. Strong $\pi-\pi$ stacking interaction of the oxyanion hole residue Trp33 with the two benzene rings of 
Phe148 and Phe200 are present throughout the simulation of the mutant system, but not of the wild type enzyme. These strong interactions are likely to keep the cap domain in a stable conformation and thus stabilize the substrate inside the binding pocket.
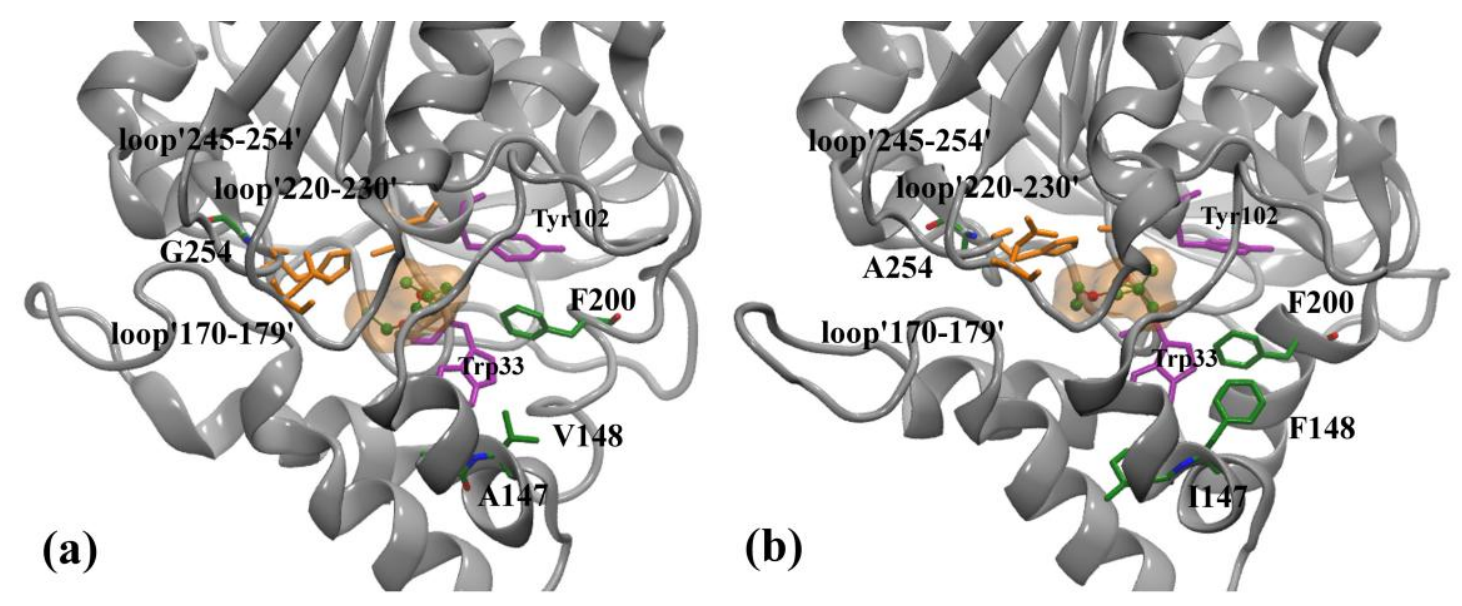

Fig. 3. An overview of the active site in $R h$ Est $_{\mathrm{WT}^{\mathrm{T}}}-\mathrm{S}-\mathrm{DmCpCe}$ (a) and

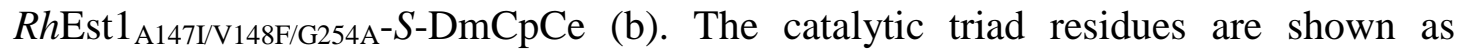
orange sticks, the oxyanion hole residues as purple sticks and the substrate $S$-DmCpCe in an orange surface representation.

As discussed above, the loop "170-179" that acts like a gate for substrate entrance/product exit showed markedly different dynamics in the wild type and mutant systems (Fig. 1(b)). Unlike in the wild type RhEst1, the loop "170-179" in the mutant is in an open conformation during the entire simulation, which may facilitate the release of the product from the enzyme active site (Fig. 3 and Fig. S5). The mutation site 254 is located on the opposite side of the substrate entrance channel and near the "170-179" loop. To investigate whether and how distant mutations may 
affect the dynamics of the "170-179" loop, cross-correlation analysis was carried out to quantify the correlated fluctuations between different residue pairs averaged over the entire simulations.


Fig. 4. Cross-correlation analysis of the $\mathrm{C} \alpha$ atomic fluctuations in wild type (a) and mutant A147I/V148F/G254A (b). The color bars on the right indicate the range of the correlation strength. Residue pairs with a high level of correlated motions are shown in red/orange colors, and anti-correlated motions shown in blue/cyan colors.

According to the correlation maps shown in Fig. 4 and Fig. S6, strong correlations are observed between the loop "170-179" and the loop" $220-230$ ", and between the former and the loop "245-254" in the wild type. In contrast, these correlations are much weaker in the A147I/V148F/G254A mutant; the detailed values for the correlation strength in the two systems are listed in Tables S4 and S5. Both "220-230" and "245-254" loops are located near the product exit channel. They also harbor the catalytic residues Asp225 and His253, respectively. The pattern changes in the correlated motions of the A147I/V148F/G254A mutant have led to the conformational rearrangements of the "220-230" and "245-254" loops as well as the conformations of 
catalytic residues Asp225 and His253 (Fig. S5). The structural difference in the A147I/V148F/G254A mutant ultimately affect the catalytic environment and the substrate binding to the enzyme.

\section{Binding free energy analysis}

To obtain a more quantitative insight into the binding of $S$-DmCpCe to the wild type and mutant $R h$ Est1, binding free energies were computed using the MM-PB(GB)/SA method for 1000 snapshots retrieved from the last $25 \mathrm{~ns} \mathrm{MD}$ trajectories. Table 1 and Table S3 list the MM-PB/SA and MM-GB/SA binding energies respectively for the two $R h$ Est 1 complexes.

Table 1. Binding energies of RhEst1 in complex with $S$-DmCpCe computed using the MM-PB/SA method.

\begin{tabular}{ccc}
\hline Energy (kcal/mol $)$ & $R h \mathrm{Est}_{\mathrm{WT}}-S$-DmCpCe & $R h \mathrm{Est}_{\mathrm{A} 147 \mathrm{I} / \mathrm{V} 148 \mathrm{~F} / \mathrm{G} 254 \mathrm{~A}}-S$-DmCpCe \\
\hline$\Delta E_{\text {elec }}$ & $-7.18 \pm 1.41$ & $-12.25 \pm 0.92$ \\
$\Delta E_{\mathrm{VDW}}$ & $-25.39 \pm 2.19$ & $-26.55 \pm 2.28$ \\
$\Delta E_{\mathrm{PB}, \mathrm{elec}}$ & $17.10 \pm 1.28$ & $18.09 \pm 2.71$ \\
$\Delta E_{\text {nonpolar }}$ & $-20.28 \pm 0.52$ & $-20.62 \pm 0.21$ \\
$\Delta E_{\text {disper }}$ & $30.21 \pm 0.47$ & $30.16 \pm 0.53$ \\
$\Delta G_{\text {bind }}$ & $-5.53 \pm 3.47$ & $-11.17 \pm 3.03$ \\
\hline
\end{tabular}

Using the MM-PB/SA method, the calculated binding energy is $-11.17 \pm 3.03$ $\mathrm{kcal} / \mathrm{mol}$ for the A147I/V148F/G254A mutant, an approximately 2-fold increase compared to the wild type $(-5.53 \pm 3.47 \mathrm{kcal} / \mathrm{mol})$. Since the experimental data for $K_{\mathrm{d}}$ is not available, we have compared the calculated binding energies to the experimentally determined $K_{\mathrm{m}}$ values. We note that $K_{\mathrm{m}}$ is in general different from the 
binding affinity $K_{\mathrm{d}}$. However, $R h$ Est1 is a relatively slow enzyme, primarily limited by slow $k_{\text {cat }}$, and it can thus be assumed that substrate binding is in rapid equilibrium and $k_{-1}$ is much greater than $k_{2}$, which justifies the use of $K_{\mathrm{m}}$ in place of $K_{\mathrm{d}}$. The overall trend of the computed binding free energies is consist with the experimentally determined $K_{\mathrm{m}}$ values $(0.43 \mathrm{mM}$ in the wild type versus $0.077 \mathrm{mM}$ in the A147I/V148F/G254A mutant) as listed in Table 2, indicating that the substrate binds more strongly in the mutant than in WT. The decomposition of the total binding energy reveals that the van der Waals interaction $\left(\Delta E_{\mathrm{VDW}}\right)$ contributes the most to the total binding energy $\left(\Delta G_{\text {bind }}\right)$. It is more negative in the mutant than in WT, suggesting that $S$-DmCpCe makes more extensive contacts with the mutant $R h E s t 1$ than with the WT. However, the Columbic interaction $\left(\Delta E_{\text {elec }}\right)$ appears to dictate the differences between the wild type and the triple mutant, although it is largely offset by the solvent polarization effect in both systems. These results are consistent with the fact suggest that the improved catalytic activity $\left(k_{\mathrm{cat}} / K_{\mathrm{m}}\right)$ of the mutant is achieved predominantly via decreasing $K_{\mathrm{m}}$, consistent with the experimental data shown in Table 2.

Table 2. Binding and kinetic parameters of various $R h E s t 1$ variants.

\begin{tabular}{lllll}
\hline Enzyme & $K_{\mathrm{m}}(\mathrm{mM})$ & $k_{\mathrm{cat}}\left(\mathrm{s}^{-1}\right)$ & $\begin{array}{l}k_{\mathrm{cat}} / K_{\mathrm{m}} \\
\left(\mathrm{mM}^{-1} \mathrm{~s}^{-1}\right)\end{array}$ & References \\
\hline$R h$ Est $1_{\mathrm{WT}}$ & 0.43 & 8.0 & 19 & Luan et al. $[14]$ \\
$R h$ Est $1_{\mathrm{A} 147 \mathrm{I} / \mathrm{V} 148 \mathrm{~F} / \mathrm{G} 254 \mathrm{~A}}$ & $0.077 \pm 0.020$ & $19.0 \pm 0.6$ & 240 & Luan et al. [14] \\
$R h$ Est $1_{\mathrm{G} 126 \mathrm{~S}}$ & $0.20 \pm 0.05$ & $5.6 \pm 0.4$ & 28 & This work \\
$R h$ Est $1_{\mathrm{R} 133 \mathrm{~L}}$ & $0.17 \pm 0.04$ & $14.0 \pm 1.0$ & 83 & This work \\
\hline
\end{tabular}




\section{Rational design and site-directed mutagenesis}

The above molecular dynamic simulations suggest a structural mechanism of how the residues located at the entrance tunnel and the loops near the catalytic triad are correlated with the enzymatic efficiency. Although several residues near the binding pocket were discussed and tested before [14], there are still several residues that have not been explored but may potentially improve the substrate binding and/or the enzyme activity. In the following, we tried to predict those potential residues based on the above MD simulation results on their structure and dynamics, also by considering their sequence conservation.

We first examined all the residues within $5 \AA$ of the substrate and located at the entrance channel or in the loops near the catalytic triad (residues at positions 35, 100, $102,126,127,128,133,140,144,170,200,228$ and 227). Those residues that were already included in the search library (residues at positions 33, 34, 100, 128, 131, 138, 140, 144, 147, 148, 166, 170, 184, 200, 227 and 228) in our previous error-prone PCR experiments have been eliminated[14]. Finally, six residues designated as Q35, Y102, G126, A127, R133 and F166 were chosen as candidate residues for further analysis, among which Y102 and R133 are located near the entrance tunnel, while the other four are situated at the distal site, as shown in Fig. 5. 


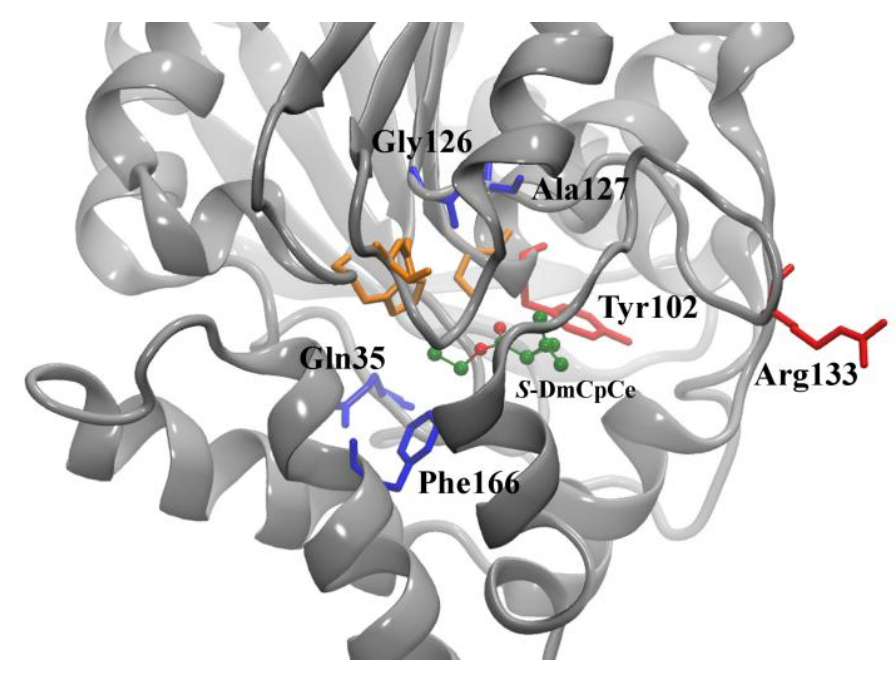

Fig. 5. The predicted "hot spot" residues located near the entrance tunnel (red) or at the opposite side of the entrance tunnel (blue), the catalytic triad residues are shown as orange sticks.

For the candidate residues Y102 and R133 located near the entrance tunnel, a smaller side chain may increase the size of the tunnel to facilitate the substrate binding. For the other residues (Q35, G126, A127, and F166) located in the loops near the catalytic triad, mutations that can rearrange the conformation of the binding site and increase the contact surface between the substrate and the enzyme may also improve the substrate binding. To refine these selections, we further considered the conservation of individual residues in the RhEst 1 family by performing a multiple sequence alignment of $R h$ Est1 and other haloperoxidases from six different organisms, as shown in Fig. S7. The "SuSPect" online server was further applied to predict how likely a single amino acid variation will affect protein stability and enzyme activity $[18,31]$. The potentially favorable mutation for each candidate residue was predicted using a support vector machine (SVM) model that was trained with 77 sequence-, structure- and systems biology-based features. As listed in Table 
S6 the predicted results suggest that Leu is more favorable than Gln at position 35; Met more favorable than Tyr at position 102; Asp more favorable than Gly at position 126; Thr more favorable than Ala at position 127; Leu is more conserved than Arg at position 133 and Phe at position 166.

Overall, we predicted six mutations to be most promising, including Q35L, Y102M, G126S, A127T, R133L and F166L. To verify the binding and catalytic activity of these predictions, site-directed mutagenesis and biochemical assays were performed for each mutant enzyme. From the results of specific activity assay shown in Table S7, it is clear that the G126S and R133L mutants indeed exhibit slightly improved enzymatic activities towards the $S$-DmCpCe substrate than the wild type enzyme.

Kinetic parameters of the purified G126S and R133L mutant enzymes were also determined experimentally, shown in Table 2 . The overall catalytic activity of both G126S and R133L mutants is improved over that of the WT. The substrate binds a little more strongly in the G126S mutant $\left(K_{\mathrm{m}}=0.20 \mathrm{mM}\right)$ than in the WT $\left(K_{\mathrm{m}}=0.43\right.$ $\mathrm{mM})$, while its turnover rate $\left(k_{\mathrm{cat}}=5.6 \mathrm{~s}^{-1}\right)$ has decreased slightly compared with the WT $\left(k_{\mathrm{cat}}=8.0 \mathrm{~s}^{-1}\right)$. Taken together, the overall catalytic efficiency $\left(k_{\mathrm{cat}} / K_{\mathrm{m}}\right)$ of G126S has increased by about 1.5 fold compared with the WT. By contrast, R133L shows both increased binding $\left(K_{\mathrm{m}}=0.17 \mathrm{mM}\right)$ and turnover rate $\left(k_{\mathrm{cat}}=14.0 \mathrm{~s}^{-1}\right)$, resulting in a 3.4 fold increase in the catalytic efficiency than the WT. Although the overall activity of none of the single mutants is better than the triple mutant, it is remarkable that the single mutant R133L shows comparable $k_{\text {cat }}$ as the A147I/V148F/G254A triple mutant, especially given the fact that R133 is not located at the active site. A 
combined mutagenesis of multiple residues may enhance the catalytic efficiency further, which is currently under investigation in our lab.

\section{Discussion}

Structure-based rational design has become a very useful strategy for protein engineering, with smaller search libraries and thus less screening cost compared with traditional experimental methods. After identifying the important mutations that may affect the enzymatic activity, site-directed mutagenesis experiment can be performed to obtain improved mutant strains with higher catalytic efficiency and suitability for industrial applications.

Using this strategy, molecular dynamic simulations were performed to reveal the detailed substrate binding mechanisms of a newly discovered recombinant esterase RhEst1. Based on the insights obtained from the simulations, we further attempted to rationally modify the enzyme to improve its activity towards the biocatalytic synthesis of S-DmCpCe. Two predicted mutants G126S and R133L indeed show modestly improved catalytic efficiency compared with the wild type enzyme, albeit through different mechanisms.

Overall, our computational work provides molecular-level insights into the structure and dynamics of the esterase $R h E s t 1$, which will facilitate future experimental efforts toward developing novel and cost-effective $R h$ Est 1 variants for industrial usage. We note that the substrate's binding affinity $\left(K_{\mathrm{m}}\right)$ is already relatively high for esterases, so the increase of the turnover rate $k_{\text {cat }}$ should be a more viable route to further improve enzyme's activity. This will be investigated in our future work. 


\section{Acknowledgements}

This work was supported by The Fundamental Research Funds for the Central Universities (Grant No. 22A201514041), National Natural Science Foundation of China (Nos. 21276082 and 31500592), and Ministry of Science and Technology, China (Nos. 2011CB710800 and 2011AA02A210). X.C. is partially supported by Computer Science and Mathematics Division at the Oak Ridge National Laboratory, which is managed by UT-Battelle, LLC under US DOE Contract No. De-AC05-00OR22725.

We would like to thank State Key Laboratory of Bioreactor Engineering at East China University of Science and Technology for the support of computing resources.

\section{References}

[1] F.M. Kahan, H. Kropp, J.G. Sundelof, and J. Birnbaum. Thienamycin: development of imipenen-cilastatin. J. Antimicrob. Chemother. 12 Suppl D (1983) 1-35.

[2] K.M. Papp-Wallace, A. Endimiani, M.A. Taracila, and R.A. Bonomo. Carbapenems: past, present, and future. Antimicrob. Agents Chemother. 55 (2011) 4943-4960.

[3] Y. Wu, K. Chen, Z. Shi , and Q. Wang. A Retrospective Study on the Incidence of Seizures Among Neurosurgical Patients who Treated with Imipenem/Cilastatin or Meropenem. Curr. Pharm. Biotechnol. 6 (2014) 685-690.

[4] W.A. Donaldson. Synthesis of cyclopropane containing natural products. Tetrahedron 57 (2001) 8589-8627.

[5] D.Y. Chen, R.H. Pouwer, and J.A. Richard. Recent advances in the total synthesis of cyclopropane-containing natural products. Chem. Soc. Rev. 41 (2012) 4631-4642.

[6] K. Chen, and F.H. Arnold. Tuning the activity of an enzyme for unusual environments: sequential random mutagenesis of subtilisin E for catalysis in dimethylformamide. Proc. Natl. Acad. Sci. U.S.A. 90 (1993) 5618-5622.

[7] W.P. Stemmer. Rapid evolution of a protein in vitro by DNA shuffling. Nature 370 (1994) 389-391. 
[8] E. Kienle, E. Senis, K. Borner, D. Niopek, E. Wiedtke, S. Grosse, et al. Engineering and evolution of synthetic adeno-associated virus (AAV) gene therapy vectors via DNA family shuffling. J. Vis. Exp. (2012) doi:10.3791/3819.

[9] F.-y. Liang, J. Huang, J.-y. He , and P. Wang. Improved enantioselective hydrolysis of racemic ethyl-2,2-dimethylcyclopropanecarboxylate catalyzed by modified Novozyme 435. Biotechnol. Bioproc. E. 17 (2012) 952-958.

[10] F. Ren, P. Wang, J. Huang, and J. He. Enzymatic Resolution of Racemic Ethyl-2,2Dimethylcyclopropanecarboxylate To S-(+)-2,2- Dimethylcyclopropanecarboxylic Acid in a Polar Organic Solvent-Water Medium. Biotechnol. Biotech. Eq. 26 (2012) 3412-3417.

[11] S.-J. Yeom, H.-J. Kim , and D.-K. Oh. Enantioselective production of 2,2-dimethylcyclopropane carboxylic acid from 2,2-dimethylcyclopropane carbonitrile using the nitrile hydratase and amidase of Rhodococcus erythropolis ATCC 25544. Enzyme Microb. Tech. 41 (2007) 842-848.

[12] C.H. Liu, J. Pan, Q. Ye, and J.H. Xu. Enzymatic production of Cilastatin intermediate via highly enantioselective hydrolysis of methyl (+/-)-2,2-dimethylcyclopropane carboxylate using newly isolated Rhodococcus sp. ECU1013. Appl. Microbiol. Biotechnol. 97 (2013) 7659-7667.

[13] Y. Zhang, J. Pan, Z.J. Luan, G.C. Xu, S. Park, and J.H. Xu. Cloning and characterization of a novel esterase from Rhodococcus sp. for highly enantioselective synthesis of a chiral cilastatin precursor. Appl. Environ. Microbiol. 80 (2014) 7348-7355.

[14] Z.-J. Luan, F.-L. Li, S. Dou, Q. Chen, X.-D. Kong, J. Zhou, et al. Substrate channel evolution of an esterase for the synthesis of cilastatin. Catal. Sci. Technol. 5 (2015) 2622-2629.

[15] D. Martinez, B. Cutino-Avila, E.R. Perez, C. Menendez, L. Hernandez, and A. Del Monte-Martinez. A thermostable exo-beta-fructosidase immobilised through rational design. Food Chem. 145 (2014) 826-831.

[16] M. Irani, U. Tornvall, S. Genheden, M.W. Larsen, R. Hatti-Kaul , and U. Ryde. Amino acid oxidation of Candida antarctica lipase B studied by molecular dynamics simulations and site-directed mutagenesis. Biochemistry 52 (2013) 1280-1289.

[17] X.Q. Yang, J.Y. Liu, X.C. Li, M.H. Chen, and Y.L. Zhang. Key amino acid associated with acephate detoxification by Cydia pomonella carboxylesterase based on molecular dynamics with alanine scanning and site-directed mutagenesis. J. Chem. Inf. Model. 54 (2014) 1356-1370.

[18] Q. Chen, Z.J. Luan, X. Cheng, and J.H. Xu. Molecular dynamics investigation of the substrate binding mechanism in carboxylesterase. Biochemistry 54 (2015) 1841-1848.

[19] O. Trott, and A.J. Olson. AutoDock Vina: improving the speed and accuracy of docking with a new scoring function, efficient optimization, and multithreading. J. Comput. Chem. 31 (2010) 455-461.

[20] J.C. Phillips, R. Braun, W. Wang, J. Gumbart, E. Tajkhorshid, E. Villa, et al. Scalable molecular dynamics with NAMD. J. Comput. Chem. 26 (2005) 1781-1802.

[21] V. Hornak, R. Abel, A. Okur, B. Strockbine, A. Roitberg, and C. Simmerling. Comparison of multiple Amber force fields and development of improved protein backbone parameters. Proteins 65 (2006) 712-725.

[22] D.A. Case, T.E. Cheatham, 3rd, T. Darden, H. Gohlke, R. Luo, K.M. Merz, Jr., et al. The Amber biomolecular simulation programs. J. Comput. Chem. 26 (2005) 1668-1688.

[23] M.J. Frisch, G.W. Trucks, H.B. Schlegel, G.E. Scuseria, M.A. Robb, J.R. Cheeseman, et al. Gaussian 03, revision c. 02; Gaussian. Inc., Wallingford, CT 4 (2004).

[24] J. Wang, W. Wang, P.A. Kollman, and D.A. Case. Automatic atom type and bond type perception in molecular mechanical calculations. J. Mol. Graph. Model. 25 (2006) 247-260. 
[25] W.L. Jorgensen, J. Chandrasekhar, J.D. Madura, R.W. Impey, and M.L. Klein. Comparison of simple potential functions for simulating liquid water. J. Chem. Phys. 79 (1983) 926-935.

[26] T. Darden, D. York, and L. Pedersen. Particle mesh Ewald: An N · log (N) method for Ewald sums in large systems. J. Chem. Phys. 98 (1993) 10089-10092.

[27] S. Pronk, S. Pall, R. Schulz, P. Larsson, P. Bjelkmar, R. Apostolov, et al. GROMACS 4.5: a high-throughput and highly parallel open source molecular simulation toolkit. Bioinformatics 29 (2013) 845-854.

[28] B.J. Grant, A.P. Rodrigues, K.M. ElSawy, J.A. McCammon , and L.S. Caves. Bio3d: an R package for the comparative analysis of protein structures. Bioinformatics 22 (2006) 2695-2696.

[29] B.R. Miller, T.D. McGee, J.M. Swails, N. Homeyer, H. Gohlke, and A.E. Roitberg. MMPBSA.py : An Efficient Program for End-State Free Energy Calculations. J. Chem. Theory Comput. 8 (2012) 3314-3321.

[30] W. Humphrey, A. Dalke, and K. Schulten. VMD: visual molecular dynamics. J. Mol. Graph. 14 (1996) 33-38.

[31] C.M. Yates, I. Filippis, L.A. Kelley, and M.J. Sternberg. SuSPect: enhanced prediction of single amino acid variant (SAV) phenotype using network features. J. Mol. Biol. 426 (2014) 2692-2701.

[32] F.-L. Du, H.-L. Yu, J.-H. Xu, and C.-X. Li. Enhanced limonene production by optimizing the expression of limonene biosynthesis and MEP pathway genes in E. coli. Bioresour. Bioprocess. 1 (2014) doi:10.1186/s40643-40014-40010-z.

[33] J. Pan, N.-D. Dang, G.-W. Zheng, B. Cheng, Q. Ye, and J.-H. Xu. Efficient production of I-menthol in a two-phase system with SDS using an immobilized Bacillus subtilis esterase. Bioresour. Bioprocess. 1 (2014) doi:10.1186/s40643-40014-40012-x.

[34] M. Holmquist. Alpha/Beta-hydrolase fold enzymes: structures, functions and mechanisms. Curr. Protein Pept. Sci. 1 (2000) 209-235.

[35] Y. Wei, J.A. Contreras, P. Sheffield, T. Osterlund, U. Derewenda, R.E. Kneusel, et al. Crystal structure of brefeldin A esterase, a bacterial homolog of the mammalian hormone-sensitive lipase. Nat. Struct. Biol. 6 (1999) 340-345.

[36] P. Liu, Y.F. Wang, H.E. Ewis, A.T. Abdelal, C.D. Lu, R.W. Harrison, et al. Covalent reaction intermediate revealed in crystal structure of the Geobacillus stearothermophilus carboxylesterase Est30. J. Mol. Biol. 342 (2004) 551-561.

[37] Y. Qiao, K. Han , and C.G. Zhan. Fundamental reaction pathway and free energy profile for butyrylcholinesterase-catalyzed hydrolysis of heroin. Biochemistry 52 (2013) 6467-6479.

[38] J. Liu, X. Zhao, W. Yang, and C.G. Zhan. Reaction mechanism for cocaine esterase-catalyzed hydrolyses of (+)- and (-)-cocaine: unexpected common rate-determining step. J. Phys. Chem. B 115 (2011) 5017-5025. 
